\title{
An Equivalent Time-Variant Storage Model to Harness EV Flexibility: Forecast and Aggregation
}

Pertl, Michael Gerold; Carducci, Francesco ; Tabone, Michaelangelo; Marinelli, Mattia; Kiliccote, Sila; Kara, Emre Can

Published in:

IEEE Transactions on Industrial Informatics

Link to article, DOI:

10.1109/TII.2018.2865433

Publication date:

2018

Document Version

Peer reviewed version

Link back to DTU Orbit

Citation (APA):

Pertl, M. G., Carducci, F., Tabone, M., Marinelli, M., Kiliccote, S., \& Kara, E. C. (2018). An Equivalent TimeVariant Storage Model to Harness EV Flexibility: Forecast and Aggregation. IEEE Transactions on Industrial Informatics, 15(4), 1899 - 1910. https://doi.org/10.1109/TII.2018.2865433

\section{General rights}

Copyright and moral rights for the publications made accessible in the public portal are retained by the authors and/or other copyright owners and it is a condition of accessing publications that users recognise and abide by the legal requirements associated with these rights.

- Users may download and print one copy of any publication from the public portal for the purpose of private study or research.

- You may not further distribute the material or use it for any profit-making activity or commercial gain

- You may freely distribute the URL identifying the publication in the public portal 


\title{
An Equivalent Time-Variant Storage Model to Harness EV Flexibility: Forecast and Aggregation
}

\author{
Michael Pertl, Member, IEEE, Francesco Carducci, Michaelangelo Tabone, Member, IEEE, \\ Mattia Marinelli, Senior Member, IEEE, Sila Kiliccote, Member, IEEE, and Emre C. Kara*, Member, IEEE
}

\begin{abstract}
The demand for vehicle charging will require large investments in power distribution, transmission, and generation. However this demand is often also flexible in time, and can be actively managed to reduce the needed investments, and to better integrate renewable electricity. Harnessing this flexibility requires forecasting and controlling electric vehicle charging at thousands of stations. This paper addresses the problem of forecasting and management of the aggregate flexible demand from tens to thousands of electric vehicle supply equipment (EVSEs). First, it presents an equivalent time-variant storage model for flexible demand at an aggregation of EVSEs. The proposed model is generalizable to different markets, and also to different flexible loads. Model parameters representing multiple EVSEs can be easily aggregated by summation, and forecasted using autoregressive models. The forecastability of uncontrolled demand and storage parameters is evaluated using data from 1341 non-residential EVSEs located in Northern California. The median coefficient of variation $(\mathrm{CV})$ is as low as $24 \%$ for the forecast of uncontrolled demand at the highest aggregation and 10-15 \% for the storage parameters. The benefits of aggregation and forecastability are demonstrated using an energy arbitrage scenario. Purchasing energy day ahead is less expensive than in the real-time market, but relies on a uncertain forecast of charging availability. The results show that the forecastability significantly improves for larger aggregations. This helps the aggregator make a better forecast, and decreases the cost of charging in comparison to an uncontrolled case by $60 \%$ with respect to an oracle scenario.
\end{abstract}

Keywords-Aggregation, Data Analysis, Demand Response, Electric Vehicles, Power System Flexibility.

\section{NOMENCLATURE}

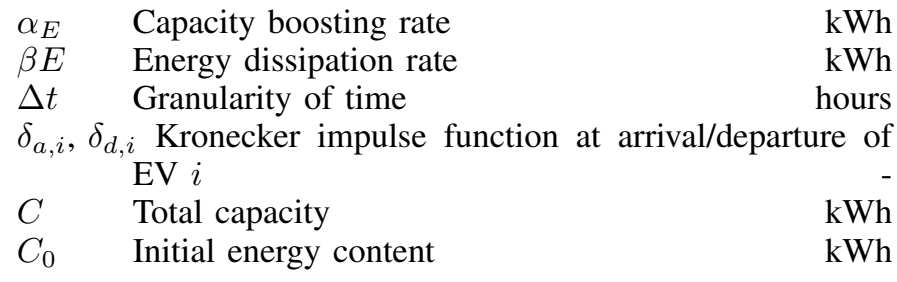

\footnotetext{
M. Pertl and M. Marinelli are with the Department of Electrical Engineering, Technical University of Denmark, Frederiksborgvej 399, 4000 Roskilde, Denmark, e-mail: \{mpertl,matm\} @elektro.dtu.dk

F. Carducci is with DIISM, Universita Politecnica delle Marche, Ancona, 60121, Italy, e-mail: francesco.carducci@pm.univpm.it

M. Tabone is with Stanford University, Stanford, CA, 94305, USA, e-mail: mtabone@stanford.edu

S. Kiliccote and E.C. Kara (* corresponding author) are with the SLAC National Accelerator Laboratory, Menlo Park, CA, 94025, USA, e-mail: \{silak,emrecan\}@slac.stanford.edu

Manuscript received February 22, 2018; revised May 19 and August 03,2018
}

$C_{a c t}$ Actual energy content

$\mathrm{kWh}$

$\operatorname{cost}_{f c}$ Cost of controlled charging with forecasted parameters

cost $_{\text {opt }}$ Cost with the optimal charging schedule \$

cost $_{u c}$ Cost of uncontrolled charging

$E_{i} \quad$ Energy to be (has been) charged into EV $i \quad \mathrm{kWh}$

$i \quad$ Indices of EV

$n \quad$ Running index

$n_{E V S E}$ number of EVSEs

$P \quad$ Charging power (decision variable) $\quad \mathrm{kW}$

$P_{i}^{+}, P_{i}^{-}$Physical limitations of $\mathrm{min} / \mathrm{max}$ charging rate of individual EVSEs $\mathrm{kW}$

$P_{\text {act }}$ Charging/discharging rate $\mathrm{kW}$

$p_{D A} \quad$ Day-ahead price $\quad \$ / \mathrm{MWh}$

$P_{f c} \quad$ Controlled charging power with forecasted parameters

$P_{\min }, P_{\max }$ Minimum/maximum charging rate of all connected Evs $\mathrm{kW}$

$P_{o p t} \quad$ Optimal charging power with perfect forecast $\mathrm{kW}$

$p_{R T} \quad$ Real-time price $\quad \$ / \mathrm{MWh}$

$P_{u c} \quad$ Uncontrolled charging power $\quad \mathrm{kW}$

$S O C$ State-of-charge of the equivalent storage -

$t \quad$ Time

$\phi \quad$ Coefficients of the AR model

$k \quad$ Constant of the AR model

$p \quad$ Order of the AR model

$s \quad$ Seasonality of the AR model

D Order of differencing of the AR model

$T \quad$ Length (datapoints) of the discrete signal

$y \quad$ Discrete signal

$\hat{y} \quad$ Forecast of the discrete signal

$C V \quad$ Coefficient of variation

$M A E$ Mean absolute error

$R M S E$ Root mean squared error

\section{INTRODUCTION}

By the end of 2015, 1.26 million electric cars were on the road worldwide. The global Clean Energy Ministerial's Electric Vehicles Initiative (EVI) 20 by 20 target calls for 20 million electric vehicles (EVs) by 2020 globally. The Paris declaration on Electro-Mobility and Climate Change and Call to Action sets a global goal of 100 million electric cars and 400 million electric 2- and 3-wheelers by 2030 [1]. Substantial market growth is needed to increase the current EV population of 1.26 million towards these ambitious targets.

From an infrastructural point of view, this rapid growth of EVs introduces several challenges. For example, in distribution 
systems, uncontrolled EV charging increases demand at some nodes, potentially exceeding the capacity of existing infrastructure, impeding voltage control, and offsetting the balance between phases [2], [3]. In China, peak load from EVs in 2030 is expected to be $479 \mathrm{GW}$ which corresponds to $54.8 \%$ of the installed generation capacity in 2009 [4].

On the other hand, EV charging is often flexible in time, and presents an opportunity to control the timing of demand to benefit distribution, transmission, and/or generation systems [5], [6]. Different control strategies are proposed in literature, including centralized [7], [8] and decentralized approaches [9]. This work addresses the challenges of modeling large aggregate populations of EVs to facilitate centralized control approaches. The disaggregation of the aggregate control signal to EVs is necessary for control application in real-world. The authors of [10] propose an algorithm to discretize the regulation signal into increments that can be met by switching on/off individual EVs. They show that the introduced error of the discretization decreases for larger numbers of EVs. In [11], the authors use an estimation of distribution algorithm (EDA) to intelligently allocate the power to the EVs. The authors of [12] propose a prioritization of the charging/discharging power based on the SOC of the individual EVs. A multi-layer realtime algorithm that assigns charging priorities to EVs based on the SOC and their departure time is proposed in [13].

The aggregation of EVSEs is necessary for several reasons. To participate in a market, a minimum resource capacity is generally required. The European EPEX spot market [14], the Nordic Nord Pool spot market [15] and the demand response (DR) programs of the California Independent System Operator (CAISO) [16] require a minimum capacity of $100 \mathrm{~kW}$. Moreover, studies show that the economical benefit and load flexibility increases by aggregating EVs [11], [17]-[19].

Aggregations EVSEs are often modeled as equivalent timevarying battery storage. The storage model is controlled to fulfill specific objectives, and serve as a flexibility asset [20], [21]. Third party aggregators or distribution system operators (DSOs) could provide ancillary services to the transmission system using the aggregate EVSEs [22]-[24]. The aggregators and DSO could also use the equivalent storage model for shortterm planning purposes in order to avoid abnormal conditions, when grid is operated close to its technical limits [25], e.g. day-ahead scheduling of demand to avoid congestion.

Many studies focus on modelling EVSE aggregations as equivalent storage systems based on several strong assumptions on the predictability of EV charging. For instance, in [26] the aggregation model needs input from the EV owner about the preferred state-of-charge (SOC) for the next day or the next hours. Then the aggregator uses this information to forecast the demand and flexibility to participate in the dayahead market. A global and divided optimization approach for participation in the day-ahead market is proposed in [27]. The global approach uses aggregated EV data but does not consider individual information. This leads to an inaccurate estimation of maximum charging power because fully charged EVs that are still plugged are not discounted in the model. In [28], the arrival and departure times of individual EVs are assumed to be known beforehand. This introduces greater uncertainties if the actual and foreseen arrival/departure times deviate from each other. In [29], the charging behavior is derived by predicting the next arrival location of EVs and the expected energy need. In [30], an exponential smoothing model is used to determine the stopover duration of EVs and from that an optimized charging schedule. A fast prediction of expected available energy and charging finishing time for individual EVSEs is presented in [31]. The authors of [32] assume that arrival times are uniformly distributed and all EVs to be charged are identical with respect to energy demand, charging session duration and maximum power consumption. The above elaborated papers suffer from great uncertainties introduced by errors in prediction/disregarding of individual EV parameters and/or strong assumptions of future EV behavior. To the best of our knowledge, no paper in the literature elaborates on the influence of aggregation on the uncertainty including the use of real EV data. In order to utilize modulation of EV charging for ancillary services or energy arbitrage, it is important to understand how well EV charging patterns can be forecasted.

A modelling approach that overcomes these drawbacks is proposed in this paper. Fig. 3 presents the proposed equivalent time-variant storage model, which is inspired by the aggregate model for thermostatically controlled loads (TCL) proposed in [33]. The model is defined by a limited number of parameters: the storage capacity $C$, the maximum/minimum charging power $P_{\max } / P_{\text {min }}$, the actual charging/discharging rate $P_{a c t}$, the current energy content of the equivalent storage $C_{a c t}$, and the energy capacity arriving/leaving the system $\alpha_{E}$ and $\beta_{E}$. The advantages of the proposed model are: a) it is applicable in different markets b) many EVSEs can be aggregated with the model by summing parameters, c) storage parameters can be forecast by using conventional time series methods such as autoregressive models without the need for more advanced algorithms, d) there is no need for information about the exact arrival and departure times in the future, e) it supports aggregating EVSEs of different charging levels.

Along with the mathematical framework that presents a novel approach to EVSE aggregation, the following analyses are presented in this paper: i) the impact of aggregation on the forecastability of the uncontrolled demand and storage parameters, ii) application of the equivalent storage model to show the benefit of aggregation in a day-ahead energy arbitrage scenario from an aggregator's perspective, and iii) method to disaggregate the aggregated charging signal to individual EVs. These analyses are key to accurate short-term planning and to enable the equivalent storage model as a flexibility asset. In line with this, the expected end user of the proposed framework is an aggregator.

The contributions of this paper are twofold: 1) description of the mathematical framework of the equivalent time-variant storage model for EVSE aggregation without assumptions on expected future driving patterns and/or exact arrival/departure times of individual EVs, 2) analysis of the size of aggregation on the forecast performance: to the best of our knowledge this paper is the first one analyzing and discussing the relation between aggregation size and uncertainty of short-term forecasts of uncontrolled demand and the proposed storage model parameters. 


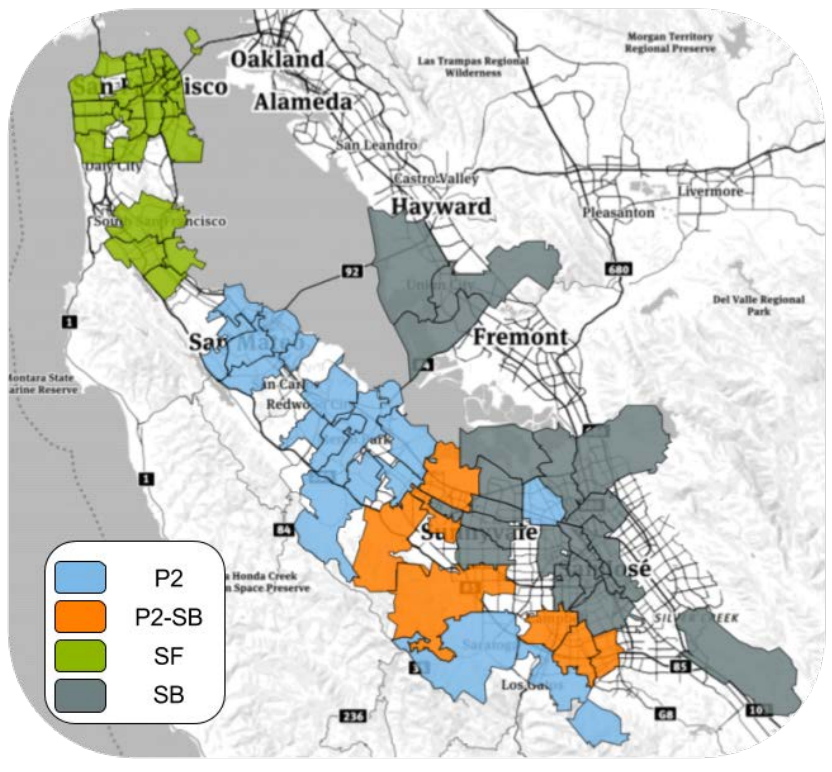

Fig. 1. Zip code regions forming the VAPs [34].

The analysis is carried out using data collected from over 1341 non-residential EVSEs, located throughout 75 zip code regions in Northern California, spanning one year. A more detailed description of the data is given in section II. The mathematical framework of the equivalent storage model is described in section III. The aggregation metric, forecasting method and forecasting results are discussed in section IV. One envisioned application of the storage model — which highlights the benefits of aggregation-is shown in section V.

\section{DATASET}

\section{A. EVSE characteristics}

The data set for this study was provided by ChargePoint. It includes data from 1341 EVSEs throughout 75 zip code regions in Northern California with 451,999 charging sessions covering the full year of 2013. For each charging session, i.e. from plug-in to departure of an EV, following data is reported:

1) Plug-in and departure time stamps

2) Average and peak power every $15 \mathrm{~min}$

3) Charged energy every $15 \mathrm{~min}$

4) Charging port type

5) Zip code

6) Non-residential building category

In order to join data from the EVSEs with local information that is identified by zip code, virtual aggregation points (VAPs) are created by aggregating EVSEs within a contiguous set of zip codes. The VAPs mostly coincide with the Pacific Gas and Electric Company's (PG\&E) sub-load aggregation points (sub-LAPs). A more detailed description of the VAPs is given in [34].

The spatial distribution of the four VAPs with the most charging sessions is shown in Figure 1. All four VAPs are adjacent and located in San Francisco Bay Area. The number
TABLE I. SUMMARY OF VAPS USED IN THIS STUDY.

\begin{tabular}{|c|c|c|c|c|}
\hline VAP & Region & $\begin{array}{r}\text { \# of zip } \\
\text { code } \\
\text { regions }\end{array}$ & $\begin{array}{r}\text { \# of } \\
\text { charging } \\
\text { sessions }\end{array}$ & $\begin{array}{r}\text { \# of } \\
\text { charging } \\
\text { sessions/day }\end{array}$ \\
\hline P2-SB & $\begin{array}{l}\text { Peninsula and } \\
\text { South Bay }\end{array}$ & 7 & 207501 & 568.50 \\
\hline SB & South Bay & 21 & 112250 & 307.53 \\
\hline SF & San Francisco & 30 & 72996 & 199.99 \\
\hline $\mathbf{P 2}$ & Peninsula & 17 & 59252 & 162.33 \\
\hline
\end{tabular}

of zip code regions that form each of the VAPs, number of charging sessions and number of charging session per day are summarized in Table I. More than $99 \%$ of the charging sessions in the dataset are from Level 2 EVSEs with a capacity between $4-7 \mathrm{~kW}$ unidirectional. About two third of the EVSEs are located at workplaces, the rest is distributed over hospitals, parking areas, universities and airports.

\section{B. Data Preprocessing}

The dataset is preprocessed before the analysis. Charging sessions with an energy less than $0.1 \mathrm{kWh}$ are removed. Time stamps of charging sessions are checked to sort out simultaneous charging sessions of the same EVSE due to time stamp errors. The energy of every interval is crosschecked with the corresponding charging power of the interval, when mismatching, the session is sorted out. The EV arrival times are rounded down and the EV departure times are rounded up to the nearest $15 \mathrm{~min}$ interval to harmonize the data.

\section{Qualitative Effect of Aggregation}

To highlight the effect of aggregation on the consumption pattern of EVs, Fig. 2 shows a time-domain plot of different EV aggregation sizes for a weekly period. As expected, the higher the number of aggregated EVSEs, the less volatile the pattern becomes. This suggests that the charging demand becomes more forecastable as the size of the EVSE aggregation increases, an observation that is studied in detail in section IV-D. Weekdays show a significantly higher demand than weekends, which may be due to the fact that most of the EVSEs are located at workplaces.

\section{Equivalent Time-Variant Storage Model}

\section{A. Equivalent Storage Parameters}

A storage model can be described by a number of parameters that are related to physical quantities. The main advantage of the storage model is that all parameters can be evaluated per EVSE and easily aggregated by summation over an arbitrary number of EVSEs. The model consists of seven timedependent parameters described as follows:

$\boldsymbol{\alpha}_{\boldsymbol{E}}(\boldsymbol{t})$ in (kWh): The capacity boosting rate $\alpha_{E}$ represents the amount of capacity added to the equivalent storage at time $t$. It is proportional to the amount of capacity connecting to the system at time $t$. Equation (1) shows how $\alpha_{E}$ is aggregated for an arbitrary number of EVSEs, $n_{E V S E} . E_{i}$ represents the amount of energy to be charged during charging session of 


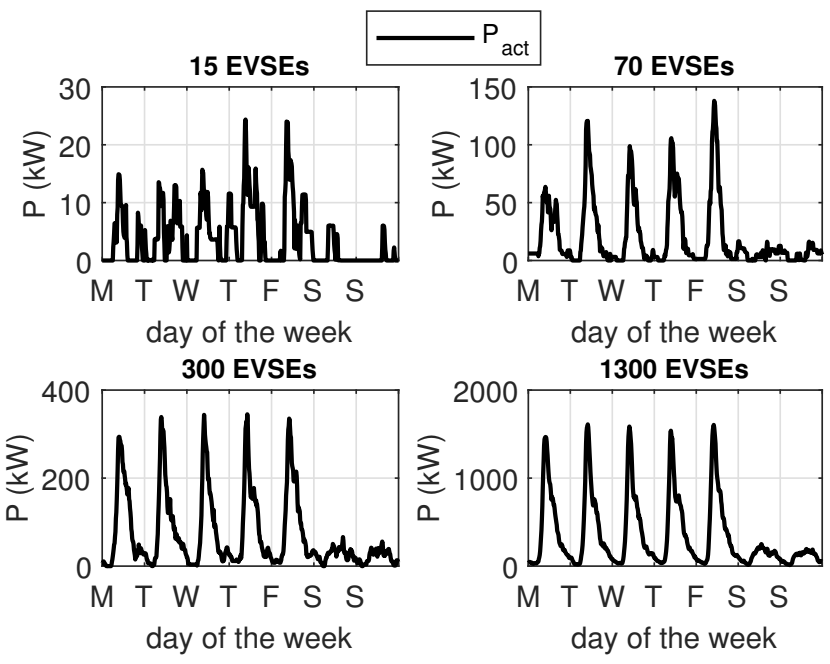

Fig. 2. Weekly demand at different aggregations.

EV $i$. Equation (2) defines $\delta_{a, i}$, which is a Kronecker impulse function centered at the arrival time of the $i$-th $\mathrm{EV}$; it assumes the value 1 during the interval that the $\mathrm{EV}$ arrives and 0 elsewhere.

The capacity added at each time is equal to the amount of energy that will be charged in initializing sessions, not the actual total capacity of EV batteries that are connecting. The latter would include any residual energy left in batteries when they plug in as well as any uncharged capacity that remains when they depart.

$$
\begin{gathered}
\alpha_{E}(t)=\sum_{i=1}^{n_{E V S E}} E_{i} \cdot \delta_{a, i}(t) \\
\delta_{a, i}(t)=\left\{\begin{array}{l}
1 \text { at arrival time of } \mathrm{EV} \mathrm{i} \\
0 \text { otherwise }
\end{array}\right.
\end{gathered}
$$

$\boldsymbol{\beta}_{\boldsymbol{E}}(\boldsymbol{t})$ in (kWh): The energy dissipation rate, $\beta_{E}$, represents the amount of energy and capacity leaving from the system when a car is unplugged. Now, $E_{i}$ represents the amount of energy that has been charged during the session, while $\delta_{d, i}$ is a Kronecker impulse function centered at the EV departure time. Note that the energy removed from the system is of equal magnitude as the added capacity when plugging in, but with opposite sign.

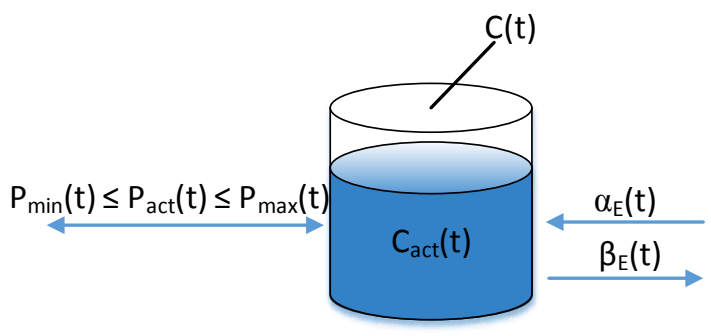

Fig. 3. Proposed equivalent time-variant storage model.

$$
\begin{gathered}
\beta_{E}(t)=\sum_{i=1}^{n_{E V S E}}-E_{i} \cdot \delta_{d, i}(t) \\
\delta_{d, i}(t)=\left\{\begin{array}{l}
1 \text { at departure time of } \mathrm{EV} \mathrm{i} \\
0 \text { otherwise }
\end{array}\right.
\end{gathered}
$$

$C(t)$ in (kWh): The total capacity of the system at time $t$ equals the sum of capacity at the previous time step and the added and removed capacity from plugging or unplugging EVs, respectively.

$$
C(t)=C(t-1)+\alpha_{E}(t)+\beta_{E}(t)
$$

$C_{a c t}(\boldsymbol{t})$ in (kWh): The state variable $C_{a c t}$ describes the actual energy content of the equivalent storage. $\Delta t$ is the granularity of time, which is 15 minutes throughout this work.

$$
C_{a c t}(t)=C_{a c t}(t-1)+P_{a c t}(t) \cdot \Delta t+\beta_{E}(t)
$$

$\boldsymbol{P}_{a c t}(\boldsymbol{t})$ in $(\mathbf{k W})$ : Charging/discharging rate of the EVs plugged into the system at time t. A positive sign represents charging while a negative sign represents discharging.

$\boldsymbol{P}_{\min }(t)$ and $\boldsymbol{P}_{\max }(t)$ in $(\mathbf{k W})$ : The minimum/ maximum charging rate of all EVs connected at time t. $P_{i}^{+}$and $P_{i}^{-}$are the physical limitations of the minimum/maximum charging rate of individual EVSEs. $P_{i}^{-}$can be negative if the EVSEs have vehicle-to-grid (V2G) capability.

$$
\begin{aligned}
P_{\text {min }}(t)=P_{\min }(t-1)+\sum_{i=1}^{n_{E V S E}} P_{i}^{-} \cdot \delta_{a, i}(t)+ & \\
& \sum_{i=1}^{n_{E V S E}}-P_{i}^{-} \cdot \delta_{d, i}(t)
\end{aligned}
$$

$$
\begin{aligned}
P_{\max }(t)=P_{\max }(t-1)+ & \sum_{i=1}^{n_{E V S E}} P_{i}^{+} \cdot \delta_{a, i}(t)+ \\
& \sum_{i=1}^{n_{E V S E}}-P_{i}^{+} \cdot \delta_{d, i}(t)
\end{aligned}
$$

$S O C(t)$ in (\%): The state-of-charge (SOC) of the storage is derived from the other parameters and the state variable. It is defined according to the general convention as the actual energy content of the equivalent storage $C_{a c t}(t)$ divided by the total capacity $C(t)$. In the case that the total capacity is zero, we define the SOC to be zero.

$$
S O C(t)=\left\{\begin{array}{l}
\frac{C_{a c t}(t)}{C(t)} \cdot 100 \% \text { for } C(t) \neq 0 \\
0 \text { else }
\end{array}\right.
$$

The minimum charging/discharging power of the EVSEs can be set to a negative number corresponding to the V2G capability of the EVSE. However, if V2G is available, additional considerations around the assumption that there is no residual energy left in the battery when the EV is connected, shall be made.

In a V2G scenario with unknown residual energy storage capacity, only the energy that has been charged into the battery can be used for V2G operation. A constraint for the minimum SOC at the end of charging session needs to be defined. The resulting costs may be lower than in a pure charging scenario. From a theoretical standpoint, the optimum may be found only 
if the residual energy storage capacity is known when an EV connects. While the proposed storage model does allow for V2G capability, we consider assessments of the value of V2G as beyond the scope of this paper.

One of the key advantages of the proposed model is that no information about exact arrival and departure times of the EVs in the future is needed. The storage parameters $\boldsymbol{\alpha}_{\boldsymbol{E}}, \boldsymbol{\beta}_{\boldsymbol{E}}$, $\boldsymbol{P}_{\boldsymbol{m i n}}$ and $\boldsymbol{P}_{\boldsymbol{m a x}}$ include the exact arrival and departure times, but this is only the case for the calculation with historical information before the forecasting step. The forecast of the storage parameters is exclusively based on autoregressive models which take the beforehand calculated time series of parameters based on historical data as input. Hence, the arrival and departure times in the future are implicitly included in the aggregated forecasted parameters but they are not explicitly needed.

\section{B. Example Storage Parameter Evolution over Time}

For better understanding of the storage parameters, Fig. 4 shows an example of the storage parameter evolution over a 24 hour period for two EVSEs. The example is taken from original measurement data and presents current practice of uncontrolled charging. The top subplot shows the evolution of the total/actual storage capacity and the added or removed capacity of the system during arrivals and departures of EVs. At $t=5.45$ the first $\mathrm{EV}$ arrives and adds about $20 \mathrm{kWh}$ to the system, i.e. the total storage capacity at that time is equal to $\alpha_{E}$ as the capacity is a function of the previous capacity plus $\alpha_{E}$ and $\beta_{E}$ as shown in (5). At the same time the maximum charging rate of the storage increases from zero to $6.6 \mathrm{~kW}$ (level 2 EVSE) as seen in the middle subplot. The EVSEs do not to have vehicle-to-grid (V2G) capability, hence, the minimum charging power is zero as no discharging is possible. The EV immediately starts to charge with about $5 \mathrm{~kW}$ after plugged to the EVSE. At 06.45 a second EV arrives and plugs to another EVSE. The second EV's added capacity $\alpha_{E}$ is larger than the one of the first EV. The total capacity increases and is now the sum of the two connected EVs. The maximum power increases to $13.2 \mathrm{~kW}$ as the other EVSE is also of level 2 type. Also the second car starts immediately to charge and $P_{\text {act }}$ increases to about $10 \mathrm{~kW}$. The SOC drops as the second EV connects as the total capacity suddenly increases while the actual energy continuously increases as the cars are being charged at a finite rate. After connection of the second EV, both EVs charge until full, sometime between 12.00 and 13.00. At 13.30 the first EVSE leaves and the storage capacity is reduced by the same amount that was added at 05.45 , hence, the total capacity is equal to the added capacity of the second EV. However, no charging happens anymore as the EV has already been fully charged. The second EV leaves at 17.30 and all parameters drop to zero as no EV is connected anymore.

\section{InFLUENCE OF AgGREGATION ON SHORT-TERM FORECASTABILITY}

\section{A. Aggregation Metric}

A metric which allows comparison of aggregations on a common basis needs to be established because EVSEs are
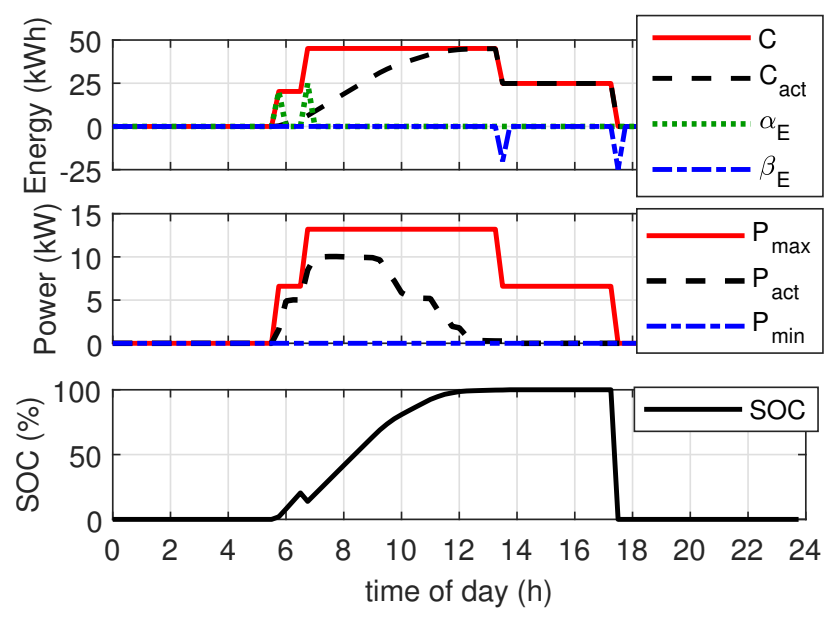

Fig. 4. Storage parameter evolution for two EVSEs over $24 \mathrm{~h}$ period.

visited at different frequency, i.e. some EVSEs have a higher demand than others. Hence, the number of EVSEs is not a suitable aggregation metric for comparison because the energy demand differs among them. Therefore, it is proposed to aggregate EVSEs by the average daily energy demand over the past two weeks (see (10)) before the forecasting period in order to compare aggregations with similar energy demand. For example, an aggregation of $100 \mathrm{kWh}$ can be achieved by a low number of EVSEs with many charging sessions or by a large number of EVSEs with a few charging sessions.

$$
E_{a g g}=\sum_{i=1}^{n_{E V S E}} \bar{E}_{i}^{14 d}
$$

\section{B. Autoregressive Forecast Models}

Autoregressive integrated moving average (ARIMA) models are used for short-term forecasting of the parameters. An ARIMA model is fit for each parameter using the Box-Jenkins methodology for analyzing the autocorrelation function (ACF) and partial autocorrelation function (PACF) of the input series [35]. The analysis is carried out for four parameters: $P_{a c t}$, $P_{\max }, C$ and $\alpha_{E}$. The motivation behind selection of these parameters is given in sections IV-D and IV-E.

The Box-Jenkins procedure suggested to use seasonal autoregressive (AR) models without moving average (MA) terms for all parameters. However, the models for the four parameters have different numbers of autoregressive lags and differencing.

The general AR model, including seasonality, is shown in (11). It consists of a constant $k$, model order $p$, coefficient $\phi_{i}$ of the i-th order, and seasonality $s$. The seasonality $s$ is equal to 672 data points, which corresponds to one week (96 data points/day $\cdot 7$ days $=672 /$ week). The parameter $D$ is the order of differencing of the time series. The parameters of all autoregressive models and the computation time per model are summarized in Table II.

The evolution of $\alpha_{E}$ represents a point process which is not suitable for AR models, instead, the cumulative sum of $\alpha_{E}$ is forecasted. Forecasting the cumulative sum of $\alpha_{E}$ is actually more convenient for the application presented in Section V. 


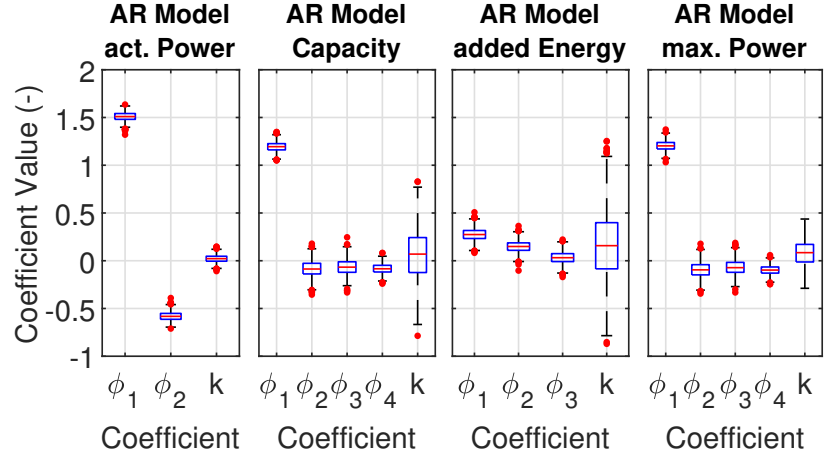

Fig. 5. Variation of AR model coefficients for 1000 EVSE combinations.

The computation times represent the time it takes to infer a model and afterwards calculate the day-ahead forecast of the parameter on a laptop with Intel i7 processor and $8 \mathrm{~GB}$ of RAM. The presented values are average values from 500 models for each parameter and it shows that inferring a model takes much longer than the forecasting.

$$
y_{(t)}=k+y_{(t-s)}+\sum_{i=1}^{p} \phi_{i}\left(y_{(t-i)}-y_{(t-i-s)}\right)
$$

TABLE II. SUMMARY OF THE AR MODEL PARAMETERS AND COMPUTATION TIME PER MODEL

\begin{tabular}{cccccc}
\hline model & \multicolumn{2}{c}{ parameter } & \multicolumn{2}{c}{ computation time (s) } \\
\hline- & $p$ & $s$ & $\mathrm{D}$ & infer & forecast \\
\hline \hline$P_{a c t}$ & 2 & 672 & 0 & 7.5 & 0.026 \\
\hline$C, P_{\max }$ & 4 & 672 & 0 & $14.5 / 12.9$ & $0.029 / 0.026$ \\
\hline$\alpha_{E}$ & 3 & 672 & 1 & 9.5 & 0.027 \\
\hline
\end{tabular}

Therefore, the coefficients of the AR models are derived for each aggregation. In order to obtain the coefficients, 20 random combinations of EVSEs are produced for each aggregation and day to be forecasted. The average of the coefficients of the 20 models yields the model parameters for the specific aggregation and day to be forecasted. The data used to generate the 20 random EVSE combinations only include 'past' data from before the forecasting period.

To justify this approach the variation of the coefficients for 1000 different combinations of EVSEs for one aggregation is shown in Fig. 5. The four subplots show the results for the four considered parameters $P_{a c t}, P_{\max }, C$ and $\alpha_{E}$. The variation of the coefficients is relatively small and therefore only the averaged model with fixed coefficients is used instead of refitting AR coefficients for every combination of EVSEs. This approach reduces the total computational effort of producing the forecast significantly.

\section{Metrics for Forecast Performance}

Several performance metrics such as Mean Absolute Error (MAE), Root Mean Squared Error (RMSE), Mean Average Percentage Error (MAPE) and Coefficient of Variation (CV) are used in the forecasting literature. The two most commonly used metrics are CV and MAPE [36]. In this work three performance metrics are used to quantify the performance of the forecast of the storage parameters, namely $\mathrm{CV}, \mathrm{MAE}$ and RMSE. For the uncontrolled demand only the $\mathrm{CV}$ is presented as it is assumed to show similar behavior as for the storage parameters. The $\mathrm{CV}$ is particularly suitable to compare time series at different scales [36], i.e. different aggregations. The $\mathrm{CV}$ is the ratio of the RMSE to the mean of the signal as shown in (12) where $y$ denotes the signal and $\hat{y}$ its forecast.

$$
\begin{gathered}
C V(y, \hat{y})=\frac{\sqrt{\frac{1}{T} \sum_{t=1}^{T}(\hat{y}(t)-y(t))^{2}}}{\frac{1}{T} \sum_{t=1}^{T} y(t)} \\
M A E(y, \hat{y})=\frac{\sum_{t=1}^{T}|\hat{y}(t)-y(t)|}{T}
\end{gathered}
$$

$$
\operatorname{RMSE}(y, \hat{y})=\sqrt{\frac{\sum_{t=1}^{T}(\hat{y}(t)-y(t))^{2}}{T}}
$$

\section{Forecastability of Uncontrolled Demand}

Even if no EV charging is able to be controlled, grid operators are interested in forecasting of charging in order to plan generation to match demand. Uncertainty in this forecasts causes grid operators to procure more expensive reserve generation to manage unexpected changes in demand.

The three upper subplots in Fig. 6 show a day-ahead forecast of the demand for a low, medium and high aggregation. Clearly, a higher aggregation improves the accuracy of the forecast significantly. The CV decreases from $70 \%$ to $46 \%$ and $21 \%$ for the medium and high aggregation, respectively.
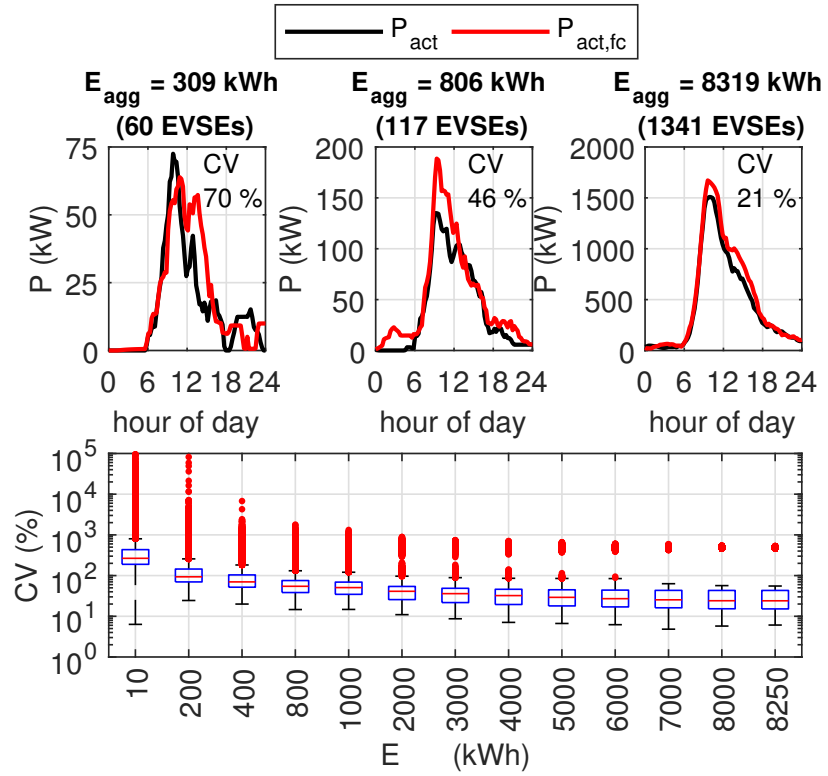

Fig. 6. Time-domain plot of forecast for three different aggregations and variation of forecast errors versus aggregation.

To further demonstrate this trend, 1000 random combinations of EVSEs for each aggregation for one month are forecasted. This totals in 30.000 random EVSE combinations for each aggregation. The performance of each forecast is evaluated by means of the $\mathrm{CV}$. 
The results of the performance assessment are shown in the lower subplot in Fig. 6. The forecast uncertainty considerably decreases with higher aggregations. The median of the forecast performance approaches about $24 \%$ whereas the $25^{\text {th }}$ and $75^{\text {th }}$ percentiles lie at $15 \%$ and $43 \%$, respectively. The results confirm the hypothesis drawn from the qualitative assessment of Fig. 2 that the demand at higher aggregations is forecastable with lower uncertainty. It is expected that the use of more advanced forecasting algorithms will further improve the performance but one should note that the basic AR models used in this analysis performed well.

\section{E. Forecastability of Storage Parameters}

The storage model, described in Section III, consists of seven parameters. Depending on the application of the model, different parameters need to be forecasted. If the storage model wants to be used as an flexibility asset for short-term planning, e.g. scheduling next day's charging pattern, the storage forming parameters $C, P_{\text {min }}, P_{\text {max }}, \alpha_{E}$ and $\beta_{E}$ need to be forecasted. In this paper, the forecasting performance of the parameters $C, P_{\max }$ and cumulative sum of $\alpha_{E}$ are analyzed. Only these three parameters are needed to run the optimization of the energy arbitrage scenario shown in Section V. The parameters $P_{\text {min }}$ and $\beta_{E}$ are not included in the analysis, but they have similar properties as their counter equivalents $P_{\max }$ and $\alpha_{E}$, respectively, i.e. the forecast performance is assumed to be in the same order of magnitude.

To determine the relationship between uncertainty of forecast and aggregation, the storage parameters for 1000 random combinations of EVSEs for each aggregation are forecasted for the next day over a period of one month $(30.000$ combinations/aggregation). The performance of each forecast is evaluated by means of the CV, MAE and RMSE.

Performance assessments of the forecasts for $\mathrm{CV}, \mathrm{MAE}$ and RMSE are shown in Fig. 7, 8 and 9, respectively. Considering the $\mathrm{CV}$, the uncertainty for all storage parameters significantly decreases for higher aggregations. The achieved performance for day-ahead forecasts for all storage parameters lies between 10 and $15 \%$ for the highest aggregation. From the results of the MAE and the RMSE it can be seen that the error increases with larger aggregations because the two metrics

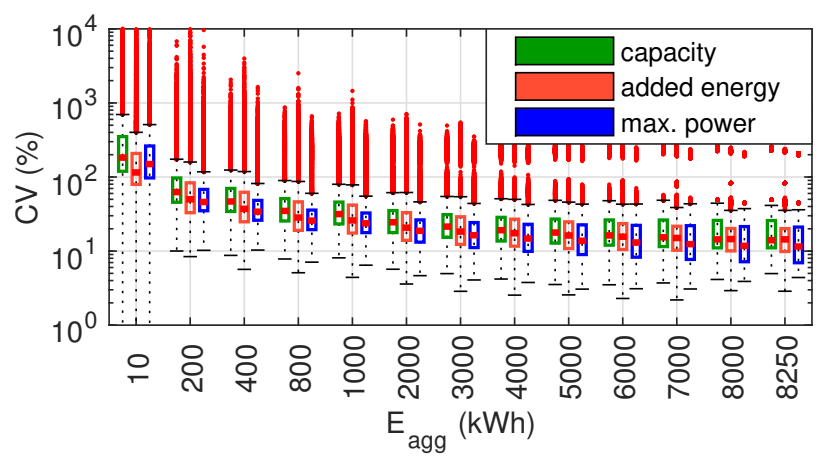

Fig. 7. $\mathrm{CV}$ of forecast versus aggregation.

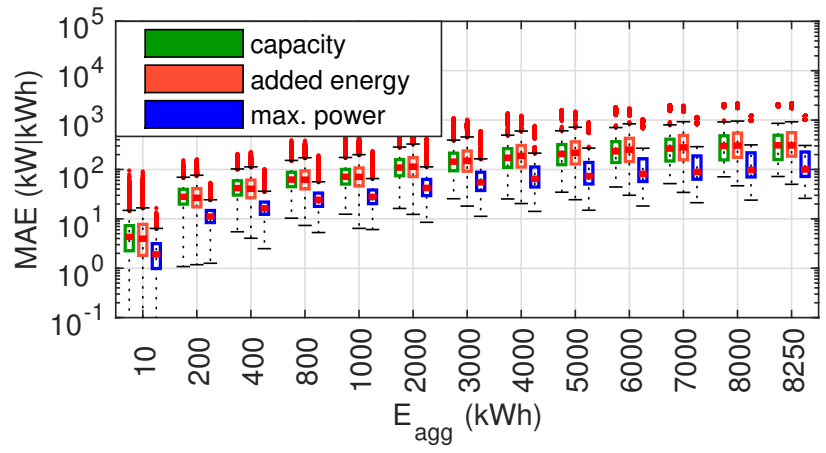

Fig. 8. MAE of forecast versus aggregation.

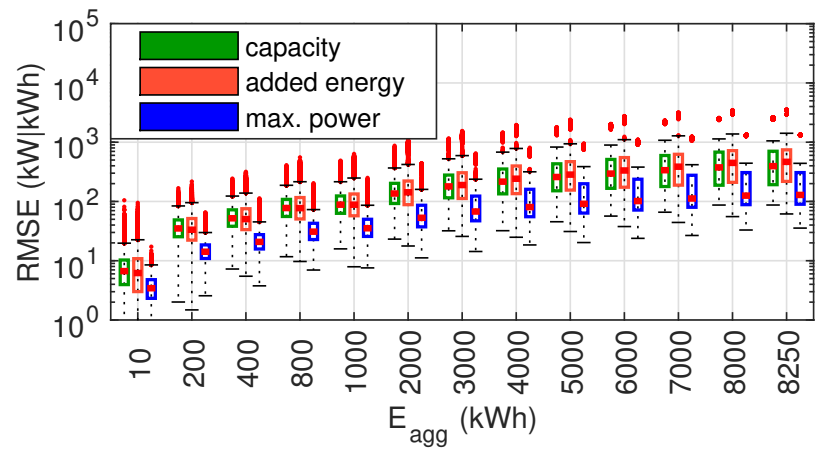

Fig. 9. RMSE of forecast versus aggregation.

do not represent percentage errors. As the signal magnitude increases also the error increases in absolute terms even though the percentage error decreases. However, MSE and RMSE show a saturation trend with increasing aggregation which allows to conclude that the forecast errors are inversely correlated with the aggregation size, i.e. relative errors reduce with larger aggregations. The plots include results for one month whereas no significant differences in the forecast performance of weekdays and weekends are observed.

\section{Application of the Storage Model}

This section presents an example application of the storage model for participation in a day-ahead market from an aggregator's perspective. The benefit of aggregation by reducing the forecast uncertainty on the costs is analyzed, i.e. how much additional costs are caused due to imperfect forecasting of the storage parameters. The storage parameters $C, P_{\max }$ and $\alpha_{E}$ are forecasted for the next day. The EVs do not have V2G capability and therefore $P_{\min }$ is equal to zero. The parameter $\beta_{E}$ is not forecasted as it is not needed for this analysis.

In order to put the application in context with the current practice, three scenarios are considered given a day-ahead price $p_{D A}$ from the CAISO day-ahead market from June 01, 2017 and a real-time price $p_{R T}$ that is arbitrarily chosen to be 1.5 times the day-ahead price.

1) uncontrolled charging $\boldsymbol{P}_{\boldsymbol{u c}}$ (current practice): the costs are determined by multiplying the uncontrolled demand with the 
real-time price as shown in (15),

2) controlled charging with forecasted storage parameters $\boldsymbol{P}_{\boldsymbol{f c}}$ (application of storage model): the power schedule is arranged, such that costs are minimized over the day-ahead price. An additional cost evaluated on the real-time price is added for deviating from the optimum due to imperfect forecast of storage parameters as shown in (16),

3) controlled charging with perfect forecast $\boldsymbol{P}_{\text {opt }}$ (benchmark case): the optimal case describes the minimal costs for the given market context due to a perfect forecast of all involved parameters and is calculated according to (17).

$$
\begin{gathered}
\operatorname{cost}_{u c}=\sum_{t=1}^{96} P_{u c}(t) \cdot p_{R T}(t) \\
\operatorname{cost}_{f c}=\sum_{t=1}^{96} P_{f c}(t) \cdot p_{D A}+ \\
\left|P_{f c}(t)-P_{o p t}(t)\right| \cdot p_{R T}(t) \\
\operatorname{cost}_{o p t}=\sum_{t=1}^{96} P_{o p t}(t) \cdot p_{D A}(t)
\end{gathered}
$$

The optimized charging schedules for 15 min intervals (96 intervals/day) for scenario 2) and 3) is derived by use of the linear optimization problem described in (18). The total costs are minimized by optimally scheduling the demand given the day-ahead price and the forecasted and original storage parameters, respectively. The day-ahead price is the locational marginal cost from one node of the CAISO day-ahead market from June 01, 2017 and the real-time price is equal to 1.5 times the day-ahead price. The first constraint describes the physical power limits within charging is possible. The second constraint describes the initial energy state of the storage. It is set to $50 \%$ of the total capacity, this value is arbitrary and can be chosen as preferred. The third constraint tracks the energy state and ensures that the energy content of the storage is between zero and the total capacity for every $15 \mathrm{~min}$ interval $\Delta t . C_{0}$ is the initial energy, $\sum_{n=1}^{t}-P_{n} \cdot \Delta t$ is the cumulative sum of the charged energy and $\sum_{n=1}^{t} \alpha_{E, n}$ is the cumulative sum of the added energy. The fourth constraint ensures that the SOC at $t_{96}$ of the storage model is again $50 \%$ as defined also for the beginning of the day and, thus, the storage model is in the same state at the beginning and at the end of the day.

The forecasted parameters are checked for certain conditions to guarantee a feasible solution in the optimization: a) $P_{\max }$ must be greater than zero at all times, b) cumulative sum of $\alpha_{E}$ must be greater or equal than the cumulative sum of the positive changes of $C$. Moreover, boundary conditions are defined, such that the storage capacity, the maximum power and the SOC at $t_{1}$ and $t_{96}$ are equal, respectively.

$$
\begin{aligned}
& \min _{P} \sum_{t=1}^{96} P(t) \cdot p_{D A}(t) \\
& \text { subject to } \\
& 0 \leq P(t) \leq P_{\max }(t) \\
& C_{0}=\frac{C\left(t_{1}\right)}{2} \\
& 0 \leq C_{0}+\sum_{n=1}^{t}\left(-P_{n} \cdot \Delta t+\alpha_{E, n}\right) \leq C(t) \\
& C_{0}+\sum_{t=1}^{96}-P(t) \cdot \Delta t+\alpha_{E}(t)=\frac{C\left(t_{96}\right)}{2}
\end{aligned}
$$

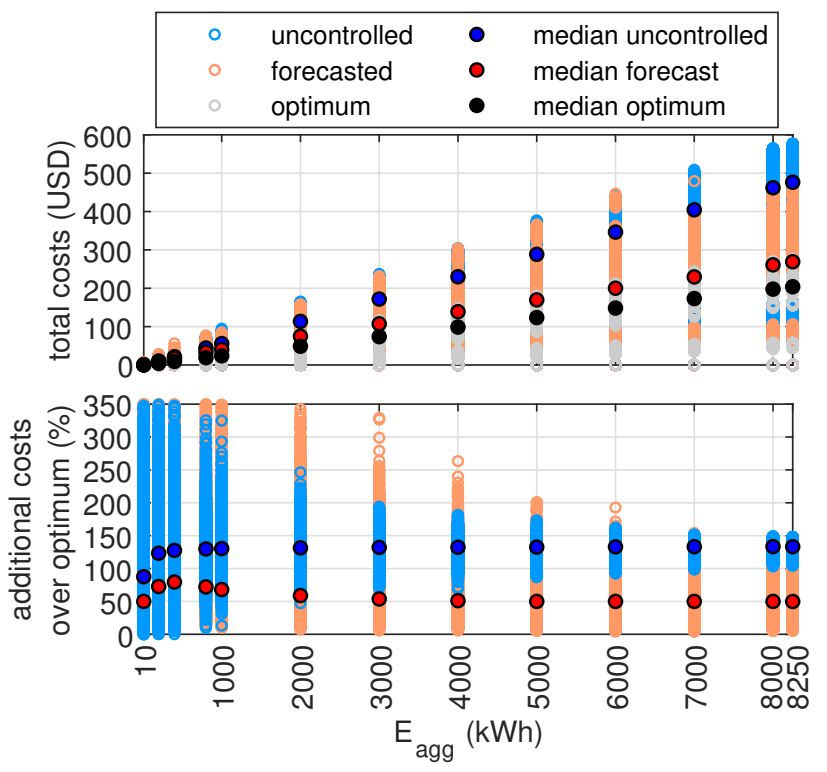

Fig. 10. Total costs and additional costs of the uncontrolled and forecasted scenario over the optimal scenario for different aggregations.

Similar to the forecastability assessment in sections IV-D and IV-E, 1000 random EVSE combinations for each aggregation are generated over a period of one month (30.000 combinations/aggregation). For each combination, the storage parameters are forecasted, an optimization is run, and the total costs for each scenario are evaluated.

The upper plot in Fig. 10 shows the total costs for each scenario for all random EVSE combinations in light and the median cost in dark colors. Obviously, the total costs are increasing with increasing aggregation. To quantify the additional costs that are added on top of the optimal case due to imperfect forecasts, the difference between the costs of the uncontrolled/forecasted scenario to the optimum are normalized over the optimum, i.e. $\left(\operatorname{cost}_{\{u c, f c\}}-\right.$ cost $\left._{\text {opt }}\right) /$ cost $_{\text {opt }}$. The lower plot shows the additional costs added to the optimum in percent of the total optimal costs.

Generally, the costs in the forecast scenario show a larger variation than the costs of the uncontrolled charging, in particular at low aggregations due to large uncertainty in the forecasts. However, the variation decreases with higher aggregations for both scenarios as forecasts become more accurate. The median cost of uncontrolled charging stabilizes at about $133 \%$ on top of the optimal costs at an aggregation of about $1000 \mathrm{kWh}$. In the forecasting scenario, the total costs only add around $50 \%$ on top of the optimal costs and is therefore more appealing from an economic perspective despite imperfect forecasts.

\section{DisAGGREGATION OF THE AGGREGATED SIGNAL TO INDIVIDUAL EVS}

The disaggregation of the aggregated charging signal is an essential aspect for application of the approach in practice. This section briefly discusses one possible disaggregation method by matching the charging schedule of the individual 
EVs with the aggregate reference signal. Two reference signals are distinguished: A) $P_{o p t}$ generated with perfect forecast, B) $P_{f c}$ generated with forecasted storage parameters.

The analysis assumes that full information from the individual charging sessions is available: arrival/departure time, energy demand etc. whereas the aggregated charging signal serves as a reference to be followed by the individual EVs. This is realized with the following optimization which minimizes the sum of absolute differences between the sum of individual charging signals and the reference charging signal:

$$
\min _{P}\left\|\sum_{t=1}^{96} \sum_{i=1}^{n_{E V S E}}\left(P_{i}(t)\right)-P_{\text {ref }}(t)\right\|
$$

subject to

$$
\begin{aligned}
& P_{i}^{-}(t) \leq P_{i}(t) \leq P_{i}^{+}(t) \\
& 0 \leq C_{0, i}+\sum_{n=1}^{t}\left(-P_{i, n} \cdot \Delta t+\alpha_{E, i, n}\right) \leq C_{i}(t)
\end{aligned}
$$

The same procedure as in the previous sections is adopted to generate random combinations of EVSEs for each aggregation. Due to computational constraints, only 100 random combinations for each aggregation over one week are generated (700 combinations for each aggregation).

Fig. 11 shows a comparison of the charging signals for one given aggregation over one day (15 min time steps). Here, the aggregation was chosen to be $6000 \mathrm{kWh}$ which is about 960 EVs.
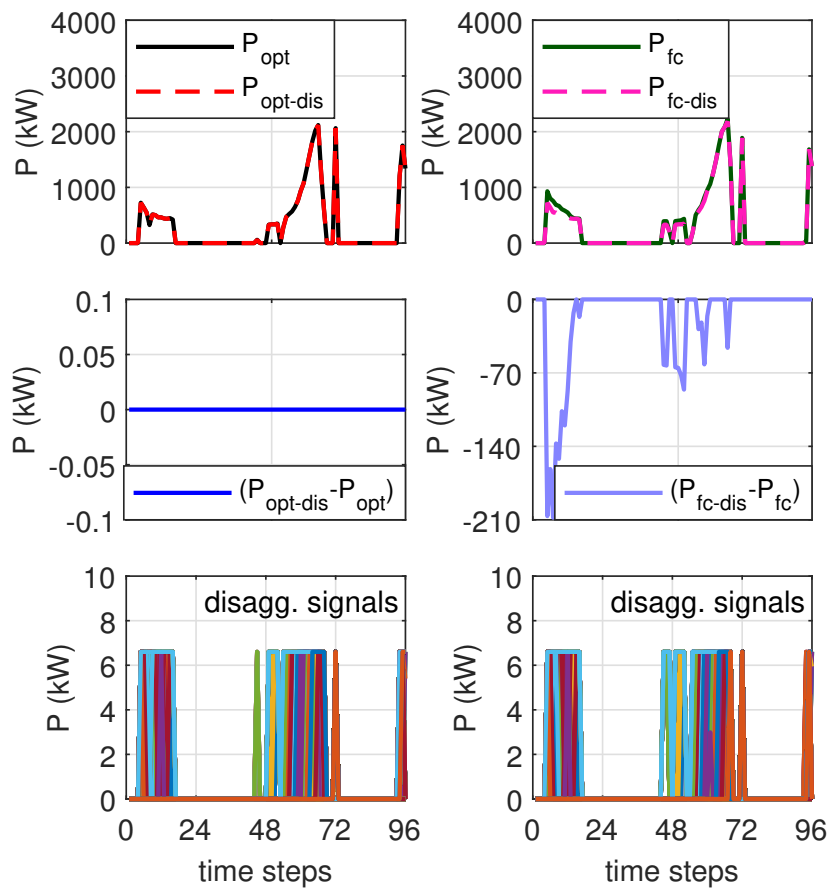

Fig. 11. Comparison of reference signal and disaggregated signal for the optimal and forecasted optimal schedule

The top left plot shows the optimal charging schedule $P_{\text {opt }}$ and the sum of the disaggregated signal $P_{\text {opt-dis }}$. The middle left plot shows the difference between the two signals. Clearly,
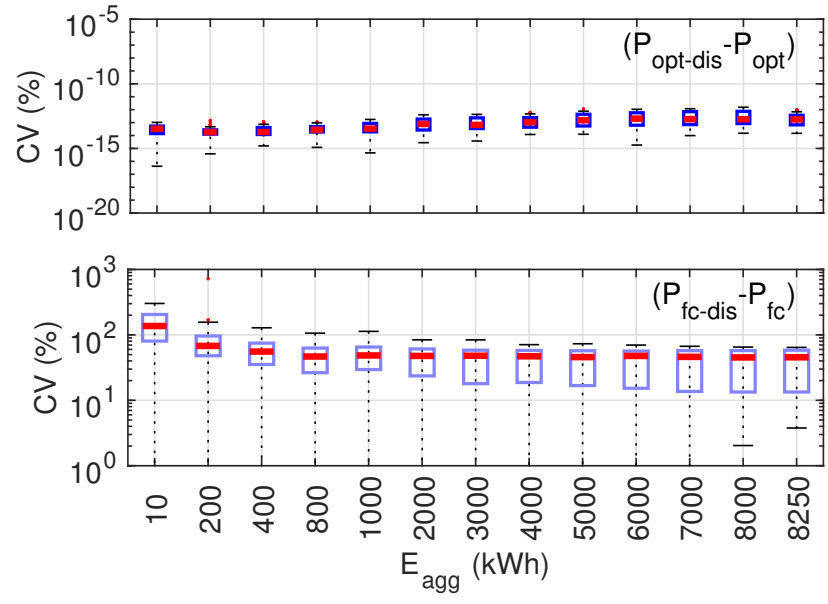

Fig. 12. $\mathrm{CV}$ of the mismatch between aggregated and disaggregated signals

there is no difference between the two signals. The disaggregated signal perfectly follows the given reference signal as the aggregated storage model is a sum of the individual parameters. Moreover, there is no forecast uncertainty involved as $P_{o p t}$ is calculated using a perfect forecast. The lower left plot shows the disaggregated individual signals, of which $P_{\text {opt-dis }}$ is comprised.

The top right plot shows the same variables but with the reference signal $P_{f c}$ that is calculated from forecasted storage parameters. Clearly, the summed disaggregated signal $P_{f c-d i s}$ does not perfectly follow the reference signal due to the uncertainty in the forecasted parameters. The middle right plot shows the difference between the two signals. Here, the forecast overestimated the demand, thus the disaggregated signal is always smaller than the reference. The lower right plot shows the disaggregated individual signals of which $P_{f c-d i s}$ is comprised. Naturally, the individual disaggregated schedules in the optimal and forecasted case are different.

In order to get insightful results and the order of magnitude of the disaggregation mismatch between the reference signal and the sum of individual signals, 100 random EVSE combinations for each aggregation over one week $(=700$ combinations/aggregation) are generated. Then the $\mathrm{CV}$ for the difference between $\left(P_{o p t-d i s}-P_{o p t}\right)$ and $\left(P_{f c-d i s}-P_{f c}\right)$ is calculated.

The upper plot in Fig. 12 shows that the difference between the optimal charging schedule and the sum of disaggregated signals is negligible (numerical errors), thus confirms preceding observation. The lower plot shows that in the forecasted case with the reference signal $P_{f c}$, the mismatch decreases at higher aggregations. For the highest aggregation, the median $\mathrm{CV}$ is about $45 \%$.

\section{CONCLUSION}

The paper presented a novel equivalent time-variant storage model for aggregation of EVs plugged to EVSEs. The proposed storage model has several advantages: a) general mathematical framework so that it is applicable in different 
market contexts, b) the storage parameters can be easily aggregated by summation and applied to any aggregation, c) the storage parameters can be forecasted by use of conventional methods such as autoregressive models without the need for complicated forecasting algorithms, d) the framework allows to release previous assumptions on the expected future driving pattern and/or exact arrival/departure times of EVs, e) within this framework, EVSEs of different charging levels can be included.

The main findings of the paper are summarized below. The analysis of the relation between aggregation and forecasting uncertainty showed that the error significantly reduces with larger aggregations. The median $\mathrm{CV}$ is as low as $24 \%$ for the forecast of uncontrolled demand at the highest aggregation. The forecast uncertainty of the storage parameters was even lower with an $\mathrm{CV}$ between 10 and $15 \%$. The results of the MAE and the RMSE showed that the absolute error increases with larger aggregations but a saturation trend with increasing aggregation allows to conclude that the forecast errors are inversely correlated with the aggregation, i.e. relative errors reduce with larger aggregations.

The benefit of aggregation was further shown by means of a practical application of the storage model for energy arbitraging under uncertainty. The costs of energy were minimized by optimizing the day-ahead schedule using (uncertain) forecasts of the storage parameters where deviations from the optimal schedule are penalized. The results show that the total costs using the proposed model in the given market context are only about $50 \%$ higher than the benchmark with perfect forecast. The costs in the uncontrolled case are about $133 \%$ higher than in the benchmark case. This shows the economic advantage of the proposed model over uncontrolled charging despite imperfect forecasts.

The disaggregation analysis showed that the aggregated charging signal can be disaggregated to individual EVs without error, when the forecast of the storage parameters are perfect, and future charging session information is available. When uncertainty is introduced to the forecasted storage parameters, the disaggregated solution deviates from the day-ahead reference signal, even though future charging session information is available.

For future work, additional uncertainty will be added considering that not all information from future charging sessions will be available. Therefore, a more thorough investigation of disaggregation strategies with incomplete information in addition to forecast uncertainties is essential. The disaggregation method, which will be applied in the future, highly depends on the availability of information, e.g. SOC, arrival/departure time, energy demand etc. Furthermore, the proposed model does not capture the human behavior under dynamic pricing conditions and this will be further investigated to evaluate the impact on the EVSE utilization rates at different times of the day.

\section{ACKNOWLEDGMENT}

We would like to thank ChargePoint for providing the data used in this study. This project is supported by the US Department of Energy SunShot initiative (SUNLAMP
0000-1756) and the TomKat Center for Sustainable Energy. SLAC National Accelerator Laboratory is operated for the US Department of Energy by Stanford University under Contract DE-AC02-76SF00515. M. Pertl and M. Marinelli acknowledge the support of the EU FP7 project ELECTRA (grant: 609687; website: electrairp.eu). We also would like to thank Charalampos Ziras from DTU for the fruitful discussions about disaggregation of aggregated charging signals to individual EVs.

\section{REFERENCES}

[1] International Energy Agency, "Global EV Outlook 2016 - Beyond one million electric cars," tech. rep., International Energy Agency, Paris, 2016

[2] M. Pertl, M. Marinelli, and H. Bindner, "Improved power quality monitoring through phasor measurement unit data interpretation," in Universities Power Engineering Conference (UPEC), (Stoke-on-Trent), p. 6,2015 .

[3] S. Martinenas, K. Knezovic, and M. Marinelli, "Management of Power Quality Issues in Low, Voltage Networks using Electric Vehicles: Experimental Validation," IEEE Transactions on Power Delivery, vol. 32, no. 2, pp. 971-979, 2017.

[4] Z. Luo, Y. Song, Z. Hu, Z. Xu, X. Yang, and K., Zhan, "Forecasting charging load of plug-in electric vehicles in China," in IEEE Power and Energy Society General Meeting, (San Diego), pp. 1-8, 2011.

[5] K. Knezović, M. Marinelli, P. Codani, and Y. Perez, "Distribution grid services and flexibility provision by electric vehicles: A review of options," in Universities Power Engineering Conference (UPEC), (Stoke-on-Trent), p. 6, 2015.

[6] M. M. N. Rezkalla, A. Zecchino, M. Pertl, and M. Marinelli, "Grid Frequency Support by Single-Phase Electric Vehicles Employing an Innovative Virtual Inertia Controller," in International Universities Power Engineering Conference (UPEC), (Coimbra), p. 6, 2016.

[7] C. Guille and G. Gross, "A conceptual framework for the vehicle-to-grid (V2G) implementation," Energy Policy, vol. 37, no. 11, pp. 4379-4390, 2009.

[8] C. Quinn, D. Zimmerle, and T. H. Bradley, "The effect of communication architecture on the availability, reliability, and economics of plug-in hybrid electric vehicle-to-grid ancillary services," Journal of Power Sources, vol. 195, no. 5, pp. 1500-1509, 2010.

[9] D. S. Callaway and I. A. Hiskens, "Achieving Controllability of Electric Loads," Proceedings of the IEEE, vol. 99, no. 1, pp. 184-199, 2011.

[10] E. Sortomme and K. W. Cheung, "Intelligent dispatch of electric vehicles performing vehicle-to-grid regulation," in IEEE International Electric Vehicle Conference (IEVC), (Greenville), 2012.

[11] W. Su and M.-y. Chow, "Performance Evaluation of an EDA-Based Large-Scale Plug-In Hybrid Electric Vehicle," IEEE Transactions on Smart Grids, vol. 3, no. 1, pp. 308-315, 2012.

[12] L. He, C. Li, Y. Cao, Z. Yu, and B. Fang, "Synergistic and priority, in Chinese Automation Congress (CAC), (Changsha), pp. 81-86, 2013.

[13] S. I. Vagropoulos, D. K. Kyriazidis, and A. G. Bakirtzis, "Real-Time Charging Management Framework for Electric Vehicle Aggregators in a Market Environment," IEEE Transactions on Smart Grid, vol. 7, no. 2, Market Environment,
pp. 948-957, 2016.

[14] EPEX SPOT SE, “Trading on EPEX Spot," 2016.

[15] Nord Pool Spot AS, "Trading Appendix 3: Product Specifications Physical Markets," 2011.

[16] J. Powers, "Implementation Overview for PDR," 2014.

[17] A. Brooks and T. Gage, "Integration of Electric Drive Vehicles with the Electric Power Grid - a New Value Stream," in 18th International Electric Vehicle Symposium and Exhibition, (Berlin), pp. 20-24, 2001.

[18] Z. Ma, D. Callaway, and I. Hiskens, "Decentralized Charging Control for Large Populations of Plug-in Electric Vehicles: Application of the Nash Certainty Equivalence Principle," in IEEE Conference on Control Applications (CCA), (Yokohama), pp. 191-195, 2010.

[19] F. Parise, M. Colombino, S. Grammatico, and J. Lygeros, "Mean field constrained charging policy for large populations of Plug-in Electric Vehicles," in IEEE Conference on Decision and Control, (Los Angeles), pp. 5101-5106, 2014.

[20] A. Y. Saber and G. K. Venayagamoorthy, "Plug-in vehicles and renewable energy sources for cost and emission reductions," IEEE Transactions on Industrial Electronics, vol. 58, no. 4, pp. 1229-1238, 2011.

[21] M. D. Galus, M. Gonzalez Vaya, T. Krause, and G. Andersson, "The role of electric vehicles in smart grids," Wiley Interdisciplinary Reviews: Energy and Environment, vol. 2, pp. 384-400, 2013. 
[22] W. Su, H. Rahimi-Eichi, W. Zeng, and M.-y. Chow, "A Survey on the Electrification of Transportation in a Smart Grid Environment," Ieee Transactions on Industrial Informatics, vol. 8, no. 1, pp. 1-10, 2012.

[23] ENTSO-E, CEDEC, GEODE, EURELECTRIC, and E. f. S. Grids, "TSO-DSO Data Management Report," tech. rep., TSO DSO data management Project Team, Brussels, 2015.

[24] M. G. Vayá, L. Baringo, T. Krause, G. Andersson, P. Almeida, F. Geth, and S. Rapoport, "EV Aggregation Models for Different Charging Scenarios," in 23rd International Conference on Electricity Distribution (CIRED), (Lyon), p. 5, 2015.

[25] J. A. Pecas Lopes, F. J. Soares, and P. M. Rocha Almeida, "Integration of electric vehicles in the electric power system: A conceptual framework for integrating electric vehicles into electric power systems is given; impacts and benefits arising from their use are discussed," Proceedings of the IEEE, vol. 99, no. 1, pp. 168-183, 2011.

[26] R. J. Bessa, F. J. Soares, J. A. Pecas Lopes, and M. A. Matos, "Models for the EV aggregation agent business," in IEEE PES PowerTech, (Trondheim), p. 8, 2011.

[27] R. J. Bessa and M. A. Matos, "Global against divided optimization for the participation of an EV aggregator in the day-ahead electricity market. Part I: Theory," Electric Power Systems Research, vol. 95, pp. 309-318, 2013.

[28] P. Sánchez-Martín, G. Sánchez, and G. Morales-España, "Direct load control decision model for aggregated EV charging points" IEEE Transactions on Power Systems, vol. 27, no. 3, pp. 1577-1584, 2012.

[29] O. Sundström, O. Corradi, and C. Binding, "Toward electric vehicle trip prediction for a charging service provider," in IEEE International Electric Vehicle Conference (IEVC), (Greenville), pp. 1-6, 2012.

[30] A. Aabrandt, P. B. Andersen, A. B. Pedersen, S. You, B. Poulsen, N. O'Connell, and J. Østergaard, "Prediction and optimization methods for electric vehicle charging schedules in the EDISON project," in IEEE PES Innovative Smart Grid Technologies (ISGT), (Washington, DC), pp. 1-7, 2012

[31] M. Majidpour, C. Qiu, P. Chu, R. Gadh, and H. R. Pota, "Fast Prediction for Sparse Time Series : Demand Forecast of EV Charging Station s for Cell Phone Applications," IEEE Transactions on Industrial Informatics, vol. 11, no. 1, pp. 242-250, 2015.

[32] D. Madjidian, M. Roozbehani, and M. A. Dahleh, "Emulating Batteries with Deferrable Energy Demand: Fundamental Trade-offs and Scheduling Policies," 2016.

[33] H. Hao, B. M. Sanandaji, K. Poolla, and T. L. Vincent, "Aggregate flexibility of thermostatically controlled loads," IEEE Transactions on Power Systems, vol. 30, no. 1, pp. 189-198, 2015.

[34] E. C. Kara, J. S. Macdonald, D. Black, M. Bérges, G. Hug, and S. Kiliccote, "Estimating the benefits of electric vehicle smart charging at non-residential locations: A data-driven approach," Applied Energy, vol. 155 , pp. 515-525, 2015.

[35] G. E. P. Box, G. M. Jenkins, and G. C. Reinsel, Time Series Analysis Forecasting and Control. New Jersey: John Wiley \& Sons, Inc., 4 ed., 2008.

[36] R. J. Hyndman, "Measuring forecast accuracy," 2014.

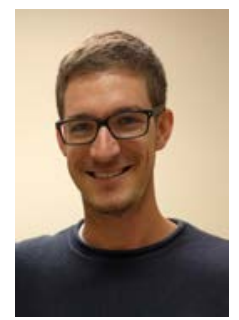

Michael Pertl (GSM'14-M'18) is a Postdoc at the Center for Electric Power and Energy (CEE) at the Technical University of Denmark (DTU). He received his BSc and Dipl.-Ing. in Electrical Engineering from the Technical University of Graz, Austria, in 2013 and 2014, respectively. He received the $\mathrm{PhD}$ degree in Electrical Engineering from DTU in 2018. His research interests include power system modelling, integration of RES and data-driven approaches applied to power systems.

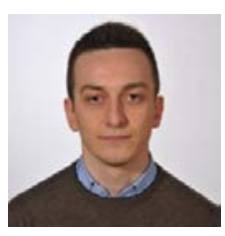

Francesco Carducci was born in Tolentino, Italy, in 1989. He received the B.S. and M.S. degree in mechanical engineering from Universita Politecnica delle Marche, Ancona, in 2012 and 2014 respectively. He is currently a Ph.D. candidate in Industrial Engineering at the Universita Politecnica delle Marche. His research interests include Smart Grid, Demand Response, Energy Storage Systems and Machine Learning.

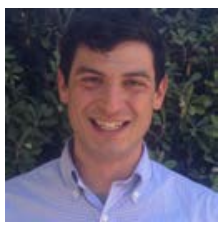

Michaelangelo Tabone (M' 14) is a Postdoctoral Fellow funded by the TomKat Center for Renewable Energy at Stanford University. He received his M.S. (12) and Ph.D. (16) from the Energy and Resources Group at the University of California, Berkeley, and received a B.S. in Chemical Engineering and a B.A. in Political Science from the University of Pittsburgh in 2010. Tabones research focuses on leveraging rapidly evolving data collection and processing techniques to promote sustainable energy systems.

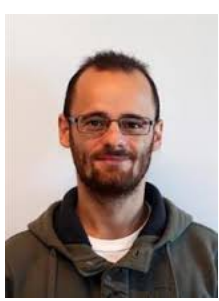

Mattia Marinelli (M'10-SM'17) was born in Genoa, Italy, in 1983. He received the B.Sc. and M.Sc. degrees in electrical engineering from the University of Genoa, in 2005 and 2007, respectively, and the European $\mathrm{Ph} . \mathrm{D}$. degree in power systems, from the same university in 2011. Since 2012, he has been with the Technical University of Denmark. His research interests include power system integration studies, wind and solar data analysis, electric vehicles, and distributed energy resources modeling.

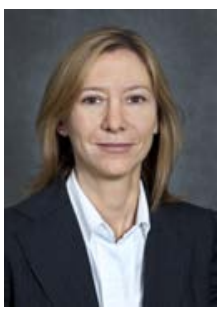

Sila Kiliccote (M' 11) is the leader of Grid Integration, Systems and Mobility (GISMo) group at SLAC National Accelerator Laboratory and Managing Director of Grid innovation at Stanford University. Her research interests include load flexibility, energy and resource management, and data science application in power systems planning and operations.

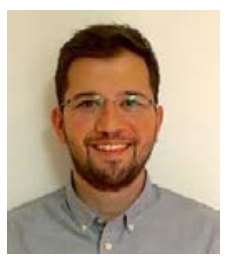

Emre C. Kara (M'14) is an Associate Staff Scientist at the Grid Integration Systems, and Mobility (GISMo) group at SLAC National Accelerator Laboratory. Prior to that, he was a Postdoctoral Researcher at Lawrence Berkeley National Laboratory (LBNL), Berkeley, CA, USA. He received his Ph.D. degree from Carnegie Mellon University, Pittsburgh, PA, USA in 2014. His research interests include datadriven modeling and control of distributed energy resources, and electrification of transportation. 